\title{
PARASITÓIDES DE LEPIDÓPTEROS MINADORES PRESENTES EM PLANTAS DE CRESCIMENTO ESPONTÂNEO EM POMAR ORGÂNICO DE CITROS EM MONTENEGRO - RS ${ }^{1}$
}

\author{
JANAÍNA PEREIRA DOS SANTOS ${ }^{2}$, FÁBIO KESSLER DAL SOGLIO $^{3}$, LUIZA RODRIGUES REDAELLI ${ }^{4}$, \\ VALMIR ANTONIO COSTA ${ }^{5}$, ESTER FOELKEL $^{6}$
}

RESUMO - Este trabalho teve como objetivos verificar se Phyllocnistis citrella ataca plantas de crescimento espontâneo, presentes em pomar de citros orgânicos, identificar nestas plantas outros lepidópteros minadores e seus parasitóides, e verificar se estes parasitóides são os mesmos relatados para P. citrella. O trabalho foi conduzido no município de Montenegro-RS, em um pomar orgânico do híbrido 'Murcott' (Citrus sinensis x C. reticulata). Realizaram-se amostragens quinzenais, de maio de 2003 a maio de 2004 , coletando-se, em cada ocasião, todas as folhas com minas contidas na área delimitada por um aro de $0,28 \mathrm{~m}^{2}$, que era jogado nas linhas e nas entrelinhas de 30 árvores sorteadas. O material coletado foi levado ao laboratório para triagem. Durante o estudo, foram registradas 11 espécies de lepidópteros minadores e 12 espécies de microimenópteros parasitóides.

Alguns gêneros identificados neste estudo já haviam sido relatados em várias regiões do mundo com espécies parasitando $P$. citrella, tais como Chrysocharis, Closterocerus, Sympiesis (Hymenoptera: Eulophidae) e Bracon (Hymenoptera: Braconidae).

Termos para indexação: Phyllocnistis citrella, controle biológico, citricultura.

\section{PARASITOIDS OF LEAFMINING LEPIDOPTERA IN SPONTANEOUS GROWTH PLANTS IN AN ORGANIC CITRUS ORCHARD IN MONTENEGRO, RS, BRAZIL}

\begin{abstract}
This work aimed to check if Phyllocnistis citrella attacks spontaneous growth plants present in an organic citrus orchard, to identify in these plants other leafmining Lepidoptera and its parasitoids, and to verify if the parasitoids species are the same ones reported for P. citrella. The work was conducted in Montenegro, RS, in an organic orchard of the hybrid 'Murcott' (Citrus sinensis x C. reticulata). Samplings were taken every other week, from May 2003 to May 2004, with a ring of $0.28 \mathrm{~m}^{2}$ being randomly thrown in the lines and interlines of 30 trees. All the plants with mines inside of the ring were collected. The screening of the material was made in laboratory. Eleven species of leafmining Lepidoptera and 12 species of microhymenopteran parasitoids were found. Some genera identified that this study had been already reported in several regions of the world with parasitizing species $P$. citrella, such as Chrysocharis, Closterocerus, Sympiesis (Hymenoptera: Eulophidae) and Bracon (Hymenoptera: Braconidae).
\end{abstract}

Index terms: Phyllocnistis citrella, biological control, citrus orchards.

\section{INTRODUÇÃO}

O Brasil destaca-se como o maior produtor mundial de frutas cítricas, com cerca de 20 milhões de toneladas na safra de 2004, numa área aproximada de 937 mil hectares. No cenário nacional, o Rio Grande do Sul é o sexto maior produtor de citros e o segundo na produção de tangerinas (IBGE, 2005).

Devido a vários fatores, como clima, solo, vegetação e a natureza perene das plantas de Citrus spp. (Rutaceae), inúmeras espécies de insetos fitófagos têm o seu desenvolvimento favorecido, podendo tornar-se pragas e comprometer a produção (Koller, 1994).

O minador-das-folhas-dos-citros, Phyllocnistis citrella (Stainton, 1856) (Lepidoptera: Gracillariidae), é uma das principais pragas da citricultura na China, Índia e Japão (Generalitat Valenciana, 1996), na Austrália, no sudeste da Ásia e no leste da
África (Heppner, 1993), e se encontra distribuído em 54 países (Cônsoli et al., 1996). O primeiro registro de P. citrella no Brasil foi em 1996, em viveiros da região de Limeira-SP (Gravena, 1996a), e, neste mesmo ano, foi constatada em pomares e viveiros do Rio Grande do Sul (Moraes et al., 1999).

As larvas de $P$. citrella têm hábito minador e atacam folhas novas das brotações de citros, fazendo galerias em forma de serpentina, de coloração prateada, provocando atrofia das folhas. Estes danos diretos reduzem a área fotossintética, uma vez que as regiões minadas ficam cloróticas ou necróticas (Schaffer et al., 1997). Além disso, pode ocorrer a necrose dos tecidos, o encarquilhamento ou abscisão das folhas (Heppner, 1993; Hoy \& Ngyuen, 1997). Phyllocnistis citrella ataca preferencialmente espécies de Citrus (Heppner, 1993), entretanto pode ovipositar em outras rutáceas (Cônsoli et al., 1996), e algumas espécies de Leguminoseae, Loranthaceae, Oleaceae e Lauraceae (Heppner,

\footnotetext{
${ }^{1}$ (Trabalho 103-2006). Recebido em 24-07-2006. Aceito para publicação em 13-02-2007.

2 Eng. Agr., M.Sc., EPAGRI, Estação Experimental de Caçador. Abílio Franco, C.P. 591, 89500-000 Caçador- SC, fone: (49) 3561 2035. E-mail: janapereira@epagri.rct-sc.br.

${ }^{3}$ Eng. Agr., PhD., UFRGS, Depto. Fitossanidade. Av. Bento Gonçalves, 7712, 91540-000 Porto Alegre-RS.

${ }^{4}$ Eng. Agr., Dr., UFRGS, Depto. Fitossanidade. Porto Alegre-RS.

${ }^{5}$ Eng. Agr., Dr., Instituto Biológico. Rod. Heitor Penteado, Km 3, 13092-543 Campinas-SP.

${ }^{6}$ Eng. Agr., Mestranda em Fitotecnia (Fitossanidade), UFRGS, Depto. Fitossanidade. Porto Alegre-RS
} 
1993). De modo geral, há pouca informação sobre o desenvolvimento completo de $P$. citrella em outros hospedeiros que não sejam Citrus.

Os primeiros trabalhos de pesquisa realizados com $P$. citrella tiveram enfoque no controle químico; entretanto, os estudos direcionaram-se para o controle biológico, pois o uso de defensivos agrícolas mostrou-se pouco eficaz e oneroso para os produtores (Gravena, 1994), devido a praga desenvolver facilmente resistência a inseticidas (Legaspi et al., 1999).

Os microimenópteros parasitóides são os principais agentes de controle biológico de $P$. citrella (Penteado-Dias et al., 1997; Mineo, 1999). De acordo com Peña et al. (1996), muitos parasitóides nativos, encontrados em áreas onde $P$. citrella se estabeleceu, apresentam elevado potencial de controle. Segundo Gravena (1996b) e Verdú (1996), estes parasitóides podem ter como hospedeiros, minadores, que atacam plantas de crescimento espontâneo presentes em cultivos próximos aos citros.

Browning \& Peña (1995) comentam que os parasitóides de $P$. citrella não são específicos e podem atacar outros lepidópteros minadores, inclusive espécies de Phyllocnistis de outras hospedeiras. Na Flórida-EUA, estes autores verificaram que diversas espécies de parasitóides nativos que passaram a atacar $P$. citrella, tinham como hospedeiros insetos minadores que se encontravam em plantas daninhas, arbustos e árvores, localizadas em áreas próximas aos pomares de citros.

As espécies de parasitóides associadas à $P$. citrella, mais comumente relatadas nas diversas regiões do mundo onde este minador ocorre, pertencem à família Eulophidae (Penteado-Dias et al., 1997), os quais, segundo Browning \& Peña (1995), apresentam muitos outros hospedeiros.

Verdú (1996) comenta que os parasitóides nativos, associados à $P$. citrella, apresentam pouca especificidade: esta, por sua vez, está relacionada ao hábitat do hospedeiro. No Brasil, são conhecidas muitas espécies nativas de parasitóides que se adaptaram e utilizam $P$. citrella como hospedeiro (Penteado-Dias et al., 1997; Costa et al., 1999; Jahnke, 2004). Provavelmente, estes parasitóides tinham outros minadores como hospedeiros, nativos ou exóticos, que viviam tanto em plantas espontâneas, quanto em plantas cultivadas.

Há poucas informações sobre o complexo de parasitóides associados a outros insetos minadores presentes na vegetação espontânea de pomares de citros. Com base no exposto, este trabalho teve como objetivos investigar a existência de algum hospedeiro de $P$. citrella dentre as plantas de crescimento espontâneo presentes em um pomar orgânico de citros, registrar a ocorrência de espécies de lepidópteros minadores e seus parasitóides nesta mesma vegetação e verificar se as espécies de parasitóides encontradas são as mesmas relatadas para $P$. citrella.

\section{MATERIAL E MÉTODOS}

O trabalho foi desenvolvido no município de Montenegro $\left(29^{\circ} 68^{\circ} \mathrm{S}\right.$ e $\left.51^{\circ} 46^{\circ} \mathrm{W}\right)$, localizado no vale do Rio Caí-RS. A topografia da região é levemente ondulada, com altitude inferior a $100 \mathrm{~m}$, pertencendo à Depressão Central. Os solos são profundos e de textura argilosa (Unidade Bom Retiro). A temperatura média anual é de $19,4^{\circ} \mathrm{C}$, apresentando chuvas abundantes ( $1.537 \mathrm{~mm} / \mathrm{ano}$ ) e bem distribuídas (Rodriguez et al., 1991).

O pomar onde o estudo foi conduzido é do tangoreiro Murcott (Citrus sinensis L. Osbeck x Citrus reticulata Blanco), enxertado sobre Poncirus trifoliata (L.) Raf, cultivado organicamente. A área é de 0,6 ha, com cerca de 370 plantas com 12 anos de idade, distribuídas no espaçamento entre plantas de $3,5 \mathrm{~m}$ e entre linhas de $5 \mathrm{~m}$.

Quinzenalmente, de maio de 2003 a maio de 2004, foram realizadas amostragens na vegetação existente no pomar. Para o sorteio dos pontos, as plantas de citros foram numeradas e, através do programa BioEstat ${ }^{\hat{a}}$ (Ayres et al., 2000), em cada ocasião, sortearam-se números que corresponderam aos pontos amostrais. Em cada ponto sorteado, recolhia-se uma unidade de amostra na linha e outra na entrelinha. Retiraram-se, em cada ocasião, 60 unidades de amostra, que consistiram de todas as plantas com minas presentes, num círculo de $0,28 \mathrm{~m}^{2}$, delimitado por um aro de PVC, com $60 \mathrm{~cm}$ de diâmetro, adaptação do método do quadrilátero proposto por Southwood (1978).

As plantas foram colocadas individualmente em sacos de plástico e transportadas até o laboratório para triagem. No Laboratório de Biologia, Ecologia e Controle Biológico de Insetos, do Departamento de Fitossanidade da Faculdade de Agronomia, UFRGS, as folhas foram examinadas com o auxílio de microscópio estereoscópio, registrando-se o número de larvas e/ou pupas de lepidópteros minadores. As folhas foram acondicionadas em placas de Petri de $9 \mathrm{~cm}$ de diâmetro e $1,5 \mathrm{~cm}$ de altura e/ou em caixas gerbox de $11,2 \mathrm{~cm}$ de diâmetro e $3,4 \mathrm{~cm}$ de altura, e mantidas em câmara climatizada (fotofase de 12 horas, $25^{\circ} \mathrm{C} \pm 1^{\circ} \mathrm{C}$ ) até a emergência dos adultos de minadores e/ou de parasitóides. Para favorecer o desenvolvimento completo dos insetos, colocou-se no pecíolo das folhas um chumaço de algodão, umedecido diariamente, para que as folhas permanecessem túrgidas.

Os parasitóides emergidos foram conservados individualmente em recipientes tipo "ependorff" contendo álcool $70 \%$, e os lepidópteros foram conservados em envelopes entomológicos. A identificação das famílias de parasitóides foi efetuada com o auxílio das chaves dicotômicas de Gauld \& Hanson (1995). Para identificação a nível genérico e específico, os parasitóides e os lepidópteros foram enviados a especialistas.

\section{RESULTADOS E DISCUSSÃO}

No pomar avaliado, nas 27 ocasiões de amostragem, não se verificou $P$. citrella nas plantas de crescimento espontâneo, porém registrou-se a emergência de 332 lepidópteros minadores, distribuídos em 11 espécies e em seis famílias (Tabela 1). Registraram-se minadores pertencentes às famílias Gracillariidae (Cameraria sp., Phyllocnistis sp., Porphyrosela sp., Lithocolletinae gen. nov.); Gelechiidae (morfoespécies 1; 2 e 3); Acrolepiidae (Antispastis xylophragma Meyrick, 1926); Nepticulidae (Stigmella sp.) e Tischeriidae (Tischeria sp.). Além destes, não foi possível identificar alguns exemplares de minadores que foram referidos como "morfoespécie nãoidentificada". 
TABELA 1 - Lepidópteros minadores e seus respectivos parasitóides, presentes em plantas de crescimento espontâneo, amostrados em pomar de 'Murcott', em Montenegro-RS (maio de 2003 a maio de 2004).

\begin{tabular}{|c|c|}
\hline $\begin{array}{l}\text { Lepidópteros minadores } \\
\text { FAMíLIA/Espécie }\end{array}$ & $\begin{array}{l}\text { Parasitíides } \\
\text { FAMíLIA: Espécie (№ de imaturos do minador parasitados pela espécie em questão) }\end{array}$ \\
\hline $\begin{array}{l}\text { GRACILLARIDAE } \\
\text { Phyllocnistis sp. } 1^{(1)}\end{array}$ & $\begin{array}{l}\text { EULOPHIDAE: Closterocerus coffeellae (141), Chrysocharis sp. } 1^{(2)}(3), \\
\text { Chrysocharis sp. } 2^{(2)}(1), \text { Chrysocharis sp. } 3(1)\end{array}$ \\
\hline & BRACONIDAE: Dolichogenidea sp. (2), Pholetesor sp. 2 (1), Orgilus sp. (1) \\
\hline Lithocolletinae gen. nov. ${ }^{(3)}$ & $\begin{array}{l}\text { EULOPHIDAE: } \text { C. coffeellae (6), Chrysocharis sp. } 2 \text { (1) } \\
\text { EUL }\end{array}$ \\
\hline ACROLEPIDAE & BRACONIDAE: Pholetesor sp. 2 (3), Stiropius sp. (2) \\
\hline Antispastis xylophragma & $\begin{array}{l}\text { EULOPHIDAE: C. coffeellae (2) } \\
\text { BRACONIDAE: Dolichogenidea sp. (9), Bracon sp. } 1 \text { (3), Bracon sp. } 2 \text { (1), } \\
\text { Pholetesor sp. } 1 \text { (1) }\end{array}$ \\
\hline $\begin{array}{l}\text { GELECHIIDAE } \\
\text { Morfoespécie } 1^{(4)}\end{array}$ & $\begin{array}{l}\text { EULOPHIDAE: C. coffeellae (13), Sympiesis sp. (2) } \\
\text { BRACONIDAE: Pholetesor sp. } 1 \text { (5) }\end{array}$ \\
\hline $\begin{array}{l}\text { Morfoespécie } 2^{(4)} \\
\text { TISCHERIIDAE } \\
\text { Tischeria Sp }\end{array}$ & EULOPHIDAE: Chrysocharisis sp. 2(1) \\
\hline Tischeria sp. & BRACONIDAE: Bracon sp. 1 (1) \\
\hline Morfoespécie não identificada $a^{(5)}$ & EULOPHIDAE: C. coffeellae (2) \\
\hline
\end{tabular}

${ }^{(4)}$ Provavelmente gênero novo ${ }^{(5)}$ Provavelmente família nova

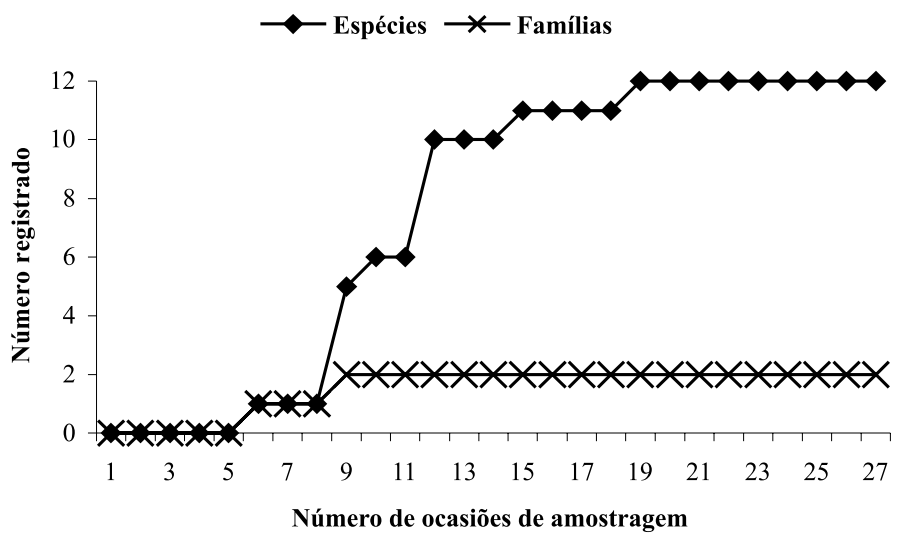

FIGURA 1- Número cumulativo de espécies e de famílias de parasitóides de lepidópteros minadores, obtidas em sucessivas amostragens em plantas de crescimento espontâneo, em pomar de 'Murcott', em Montenegro-RS (maio de 2003 a maio de 2004).

Verificaram-se parasitóides associados aos lepidópteros minadores: Phyllocnistis sp., Lithocolletinae gen. nov., A. xylophragma, Gelechiidae (morfoespécies 1 e 2), Tischeria sp. e morfoespécie não-identificada (Tabela 1). Não se observaram parasitóides associados aos minadores Cameraria sp., Porphyrosela sp., Stigmella sp. e Gelechiidae morfoespécie 3. As espécies e as famílias de parasitóides registradas refletem de fato o existente na área, pois o número de espécies estabilizouse a partir da $19^{a}$ época de amostragem (Figura 1A), e o de famílias a partir da $9^{a}$ época (Figura 1B). Associados aos lepidópteros minadores, emergiram 202 parasitóides, todos pertencentes à Hymenóptera, distribuídos em 12 espécies e em duas famílias, Braconidae e Eulophidae (Tabela 1).

Com 173 indivíduos obtidos de seis espécies de lepidópteros, os parasitóides pertencentes à família Eulophidae foram os mais numerosos. Estas espécies de parasitóides pertencem aos gêneros: Chrysocharis Förster, 1856, Closterocerus Westwood, 1833 (Entedoninae) e Sympiesis Förster, 1856 (Eulophinae) (Tabela 1).
Eulophidae possui um grande número de gêneros e espécies, com ampla gama de hospedeiros e diversidade de hábitats. De acordo com Askew \& Shaw (1986), é a família que apresenta o maior número de espécies que parasitam insetos minadores. Em geral, são endoparasitóides primários idiobiontes de insetos de várias ordens, muitos de importância agrícola (Hanson \& Gauld, 1995; Schauff et al., 1998). Em insetos minadores, atacam os estágios de larva, pré-pupa ou pupa (Browning \& Peña, 1995).

Hanson \& Gauld (1995) referem os gêneros Chrysocharis e Closterocerus como um dos principais a apresentar espécies utilizadas em programas de controle biológico de minadores. Segundo Schauff et al. (1997), as espécies de Sympiesis são ectoparasitóides idiobiontes especialistas em lepidópteros.

Neste estudo, $C$. coffeellae foi a espécie associada ao maior número de hospedeiros, (cinco), dos sete em que se registrou parasitismo. O maior número de indivíduos de $C$. coffeellae, em relação às demais espécies de parasitóides, pode ser explicado pelo fato de se tratar de um parasitóide gregário.

Braconidae foi a família em que se registrou o maior número de espécies de parasitóides, distribuídas nos gêneros: Bracon Fabricius, 1804 (Braconinae); Pholetesor Mason, 1981 (Microgastrinae); Dolichogenidea Viereck, 1911 (Microgastrinae); Orgilus Haliday, 1833 (Orgilinae) e Stiropius Cameron, 1911 (Rogadinae), perfazendo um total de 29 indivíduos emergidos de cinco espécies de lepidópteros (Tabela 1).

As espécies de Bracon estão associadas à Coleóptera, Díptera e Lepidóptera (Quicke, 1997), as de Stiropius estão associadas a Gracillariidae e Lyonetiidae minadores (Whitfield, 1988) e as de Orgilus a Gelechiidae, Coleophoridae, Oecophoridae, Psychidae, Pyralidae, Gracillariidae e Tortricidae minadores (Achterberg, 1997). De acordo com Whitfield (1997), as espécies de Pholetesor estão freqüentemente associadas a Gelechiidae minadores presentes em diversas culturas e a Gracillariidae em pomares de diferentes culturas, enquanto as espécies de Dolichogenidea são na maioria endoparasitóides solitários de várias famílias de microlepidópteros.

Espécies de alguns gêneros constatados neste estudo já foram relatadas parasitando P. citrella, tais como: Bracon sp. nas Filipinas (Baroga, 1968 apud Heppner, 1993); Chrysocharis sp. no Japão (Wu \& Tao, 1977 apud Heppner, 1993), em Honduras (Schauff et al.,1998) e na Espanha (Urbaneja et al., 1998); Sympiesis sp. na Austrália e Taiwan (Wu \& Tao, 1977 apud Heppner, 1993), nos Estados Unidos (Peña et al., 1996) e na Espanha (Urbaneja et al., 1998); Closterocerus spp. nos Estados Unidos (Browning \& Peña, 1995; Peña et al., 1996), no México, na Colômbia e em Honduras (Schauff et al., 1998).

No Brasil, apenas Sympiesis sp. já havia sido constatada parasitando P. citrella no Rio Grande do Sul (Jahnke, 2004), porém com baixa freqüência relativa $(4 \%)$.

Dentre as hipóteses que podem explicar a baixa ocorrência de parasitóides de outros lepidópteros minadores associados à P. citrella, Hoy \& Nguyen (1997) comentam que a utilização de novos recursos, como insetos-praga exóticos, por espécies nativas de parasitóides, tem sido comumente observada; entretanto, nos primeiros anos desta associação, o percentual 
de parasitismo tende a ser moderado, visto que os parasitóides nativos não estão adaptados fisiológica, etológica ou fenologicamente aos seus novos hospedeiros.

Neste trabalho, constatou-se a existência de uma considerável diversidade de espécies de lepidópteros minadores e de parasitóides associados aos mesmos. Não foi possível determinar a espécie de muitos minadores e seus parasitóides, pois além da existência de espécies novas, a falta de estudos taxonômicos de minadores, Braconidae e Eulophidae neotropicais, dentre outros, dificultou a identificação específica até mesmo das espécies já descritas.

Neste estudo, de acordo com Davis (informação pessoal), foram obtidas: uma espécie nova (Phyllocnistis sp.), um gênero novo (Lithocolletinae gen. nov.), três prováveis gêneros novos (morfoespécies de Gelechiidae) e uma nova família de minadores. Quanto aos parasitóides, segundo Hansson (informação pessoal), foram obtidas duas prováveis espécies novas de Chrysocharis.

A diversidade de organismos, neste hábitat, demonstra a importância da manutenção das plantas espontâneas nos agroecossistemas como refúgio e reservatório de agentes de controle biológico. Embora incipiente, o conhecimento dos hospedeiros preferenciais dos minadores e a época de ocorrência destes e de seus parasitóides permitem programar práticas de manejo que envolva o controle biológico, utilizando-se da vegetação de crescimento espontâneo.

Apesar de $P$. citrella não ter sido encontrada na vegetação de crescimento espontâneo, algumas das espécies de parasitóides nativos associadas aos lepidópteros minadores potencialmente poderiam vir a utilizar o minador-das-folhasdos-citros como hospedeiro, tendo em vista a nãoespecificidade de alguns deles.

\section{CONCLUSÕES}

No pomar estudado, não se encontrou $P$. citrella nas plantas de crescimento espontâneo. Entretanto, registrou-se uma grande diversidade de lepidópteros minadores e de parasitóides. Em várias regiões do mundo, espécies de alguns gêneros constatados neste estudo já haviam sido registradas parasitando P. citrella.

\section{AGRADECIMENTOS}

Ao Biólogo Luís Laux, cooperado da ECOCITRUS, por ter concedido a área de realização do estudo. Ao Dr. Donald R. Davis, do National Museum of Natural History, Smithsonian Institution (USNM), Washington DC-EUA, pela identificação dos microlepidópteros minadores. Ao Dr. Christer Hansson, do Department of Zoology, University of Lund, Suécia, pela identificação dos parasitóides Chrysocharis e Closterocerus. Ao CNPq, por conceder bolsa para o primeiro e terceiro autores, e ao Programa RS-Rural, pelo suporte financeiro.

\section{REFERÊNCIAS}

ACHTERBERG, C.V. Subfamily Orgilinae. In: WHARTON, R.A.; MARSH, P.M.; SHARKEY, M.J. (Eds). Manual of the New World genera of the family Braconidae (Hymenoptera). Washington, DC: The International Society of Hymenopterists, 1997. p. 397-402.

ASKEW, R.R.; SHAW. M.R. Parasitoid communities: their size, structure and development. In: WAAGE, J.; GREATHEAD, Y.D. (Ed.). Insect parasitoids. London: Academic Press, 1986. p. 225-264.

AYRES, M.; AYRES JÚNIOR, M.; AYRES, D.L.; SANTOS, A.S. dos. BioEstat 2.0: aplicações estatísticas nas áreas das ciências biológicas e médicas. Belém: Sociedade Civil Mamirauá; Brasília: CNPq, 2000. 272p.

BROWNING, H.W.; PEÑA, J.E. Biological control of the citrus leafminer by its native parasitoids and predators. Citrus Industry, Bartow, v.76, p. 46-48, 1995.

CÔNSOLI, F.L.; ZUCCHI, R.A.; LOPES, J.R.S. Phyllocnistis citrella Stainton, 1856 (Lepidoptera: Gracillariidae: Phyllocnistinae): a lagarta minadora dos citros. Piracicaba: FEALQ, 1996.39p.

COSTA, V.; SÁ, L.A.N. de; LASALLE, J.; NARDO, E.A.B. de; ARELLANO, F.L.; FUINI, L.C. Indigenous parasitoids (Hym.: Chalcidoidea) of Phyllocnistis citrella Stainton (Lep.: Gracillariidae) in Jaguariúna, São Paulo State, Brazil: preliminary results. Journal Appllied Entomology, Hamburg, v.123, p.237-240, 1999.

IBGE. Produção agrícola municipal: culturas temporárias e permanentes 2004. Disponível em: <http:// www.sidra.ibge.gov.br>. Acesso em: 19 dez. 2005.

GAULD, I.D.; HANSON, P.E. The evolution, classification and identification of the Hymenoptera. In: HANSON, P.E.; GAULD, I.D. The Hymenoptera of Costa Rica. Reino Unido: Oxford University Press, 1995. p.138-156.

GENERALITAT VALENCIANA. EI minador de las hojas de los cítricos (Phyllocnistis citrella St.). Valencia: Conselleria de Agricultura y Medio Ambiente, 1996. 8p.

GRAVENA, S. "Minadora das folhas dos citros": a mais nova ameaça da citricultura brasileira. Revista Laranja, Cordeirópolis, v.15, n.2, p. 397-404, 1994.

GRAVENA, S. Chega ao Brasil o minador das folhas. Informativo Agropecuário Coopercitrus, Bebedouro, v.10, n.114, p.2325, 1996a.

GRAVENA, S. Lagarta minadora dos citros no Brasil. Revista Laranja, Cordeirópolis, v. 17, n. 1, p. 286-288, 1996 b.

HANSON, P.E.; GAULD, I.D (Ed.). The Hymenoptera of Costa Rica. Reino Unido: Oxford University Press, 1995. 893p.

HEPPNER, J.B. Citrus leafminer, Phyllocnistis citrella, in Florida (Lepidoptera: Gracillariidae: Phyllocnistinae). Tropical Lepidoptera, Gainesville, v.4, n.1, p.49-64, 1993.

HOY, M.A.; NGUYEN, R. Classical biological control of the citrus leafminer Phyllocnistis citrella Stainton (Lepidoptera: Gracillariidae): theory, practice, art and science. Tropical Lepidoptera, Gainesville, v.8, n.1, p.1-19. 1997. 
JAHNKE, S.M. Parasitóides de Phyllocnistis citrella Stainton (Lepidoptera: Gracillariidae) em citros em Montenegro-RS. 2004. 103f. Tese (Doutorado em Fitotecnia: Fitossanidade)Universidade Federal do Rio Grande do Sul, Porto Alegre, 2004.

KOLLER, O.C. Citricultura: laranja, limão e tangerina. Porto Alegre: Rigel, 1994. 446p.

LEGASPI, J.C.; FRENCH, J.V.; SHAUFF, M.E.; WOOLEY, J.B. The citrus leafminer Phyllocnistis citrella (Lepidoptera: Gracillariidae) in South Texas: incidence and parasitism. Florida Entomologist, Gainesville, v.82, p.305-316, 1999.

MINEO, G. Records on indigenous antagonists of Phyllocnistis citrella (Lepidoptera: Gracillariidae) new for Italy. Bollettino di Zoologia Agraria e Bachicoltura, Torino, v.31, n.1, p.97$105,1999$.

MORAES, L.A.H. de; SOUZA, E.L. de S.; BECKER, R.F.P.; BRAUN, J. Controle químico do minador-das-folhas-doscitros Phyllocnistis citrella Stainton, 1856. Pesquisa Agropecuária Gaúcha, Porto Alegre, v.5, n.1, p.19-22, 1999.

PEÑA, J.E.; DUNCAN, R.; BROWNING, H. W. Seasonal abudance of Phyllocnistis citrella (Lepidoptera: Gracillariidae) and its parasitoids in South Florida citrus. Enviromental Entomology,Lanham, v.25, n.3, p.698-702, 1996.

PENTEADO-DIAS, A.M.; GRAVENA, S.; PAIVA, P.E.B.; PINTO, R.A. Parasitóides de Phyllocnistis citrella (Stainton) (Lepidoptera: Gracillariidae: Phyllocnistinae) no Estado de São Paulo. Revista Laranja, Cordeirópolis, v.18, n.1, p.7984, 1997.

QUICKE, D.L.J. Subfamily Braconinae. In: WHARTON, R.A.; MARSH, P.M.; SHARKEY, M.J. (Eds.). Manual of the New World genera of the family Braconidae (Hymenoptera). Washington, DC: The International Society of Hymenopterists, 1997. n.1. p.149-176.

RODRIGUEZ, O.; VIÉGAS, F.; POMPEU JÚNIOR, J.; AMARO, A.A. (Coord). Citricultura brasileira. Campinas: Fundação Cargill, 1991.492p.

SCHAFFER, B.; PEÑA, J.E.; COLLS, A.M.; HUNSBERGER, A. citrus leafminer (Lepidoptera: Gracillariidae) in lime: assessment of leaf damage and effects on photosynthesis. Crop Protection, Guildford, v.16, n.4, p.337-343, 1997.

SCHAUFF, M.E.; LASALLE, J.; COOTE, L.D. Eulophidae. In: GIBSON, G.A.P.; HUBER, J.T.; WOOLLEY, J.B. (Ed.). Annotated keys to the genera of Nearctic Chalcidoidea (Hymenoptera). Ottawa: National Research Council Research Press, 1997. p.327-429.

SCHAUFF, M.E.; LASALLE, J.; WIJESEKARA, G.A. The genera of the Chalcid parasitoids (Hymenoptera: Chalcidoidea) of citrus leafminer Phyllocnistis citrella Stainton (Lepidoptera: Gracillariidae). Journal of Natural History, London, v.32, p.1001-1056, 1998.

SOUTHWOOD, T.R.E. Ecological methods: with particular reference to the study of insect populations. London: Chapman and Hall, 1978. 524p.
URBANEJA, A.; JACAS, J.; VERDÚ, M.J.; GARRIDO, A. Dinámica e impacto de los parasitoides autóctonos de Phyllocnistis citrella Stainton, en comunidad valenciana. Investigación Agraria: producción y protección vegetales, Madrid, v.13, n.3, p.409-423, 1998.

VERDÚ, M.J. Chalcidoidea (Hymenoptera), parásitos del minador de los cítricos Phyllocnistis citrella (S) (Lep.; Gracillariidae) en España. Levante Agrícola, Valencia, v.336, p.227-230, 1996.

WHITFIELD, J.B. Revision of the Neartic species of the genus Stiropius Cameron (=Bucculatriplex Auct.) with the description of a new related genus (Hymenoptera: Braconidae). Systematic Entomology, Oxford, v.13, p.373-385, 1988.

WHITFIELD, J.B. Subfamily Microgastrinae. In: WHARTON, R.A.; MARSH, P.M.; SHARKEY, M.J. (Eds.). Manual of the New World genera of the family Braconidae (Hymenoptera). Washington, DC: The International Society of Hymenopterists, 1997. n.1, p.333-366. 